\title{
Electrical and Heat Currents in Nanoscopic System with Ferromagnetic Electrodes of Non-Collinear Magnetic Moments
}

\author{
M. WierzBiCKI* AND R. ŚWIRKOWICZ
}

Faculty of Physics, Warsaw University of Technology, Koszykowa 75, 00-662 Warsaw, Poland

Thermoelectric properties of nanoscopic system composed of a single-level quantum dot attached to ferromagnetic electrodes are studied with the use of the non-equilibrium Green function formalism. Electrical and heat currents flowing through the system under temperature gradient and bias voltage are calculated in the non-linear regime. Both charge and heat currents strongly depend on configuration of magnetic moments in the leads. TMR ratio is mostly positive, but in a region of small bias voltages, where the electron flow is determined by the temperature gradient, electrical current in parallel and antiparallel configurations flows in opposite directions leading to considerable negative TMR.

PACS: 73.23.Hk, 73.50.Lw, 85.80.Fi

\section{Introduction}

Thermoelectric properties of low dimensional systems are of fundamental and practical interest. There is a possibility to use such systems as efficient thermal-to-electric energy converters. In the linear response regime the efficiency is described by the dimensionless figure of merit ZT $=S^{2} G T / \kappa$ expressed in terms of linear thermopower $S$, electrical conductance $G$, thermal conductance and operating temperature of a device $T$. However, the measurements as well as theoretical investigations show that thermoelectric properties of the system strongly vary with temperature difference and optimal thermoelectric operation can be achieved in the non-linear, non-equilibrium regime [1-4]. These studies, up to now, have been performed for non-magnetic low dimensional systems and molecular junctions.

Recently, a great interest in thermoelectric effects and thermal efficiency of nanoscale magnetic systems can be observed due to potential applications as energy converters in spintronic devices [5-8]. Thereby, the studies of spin effects in the non-linear transport in a presence of temperature gradient are required. Non-linear thermopower as a function of temperature difference determined under the condition of vanishing currents in each spin channel was investigated for a two-level quantum dot (QD) in Ref. [9] and spin-dependent voltage was found.

Here, we discuss the system composed of a single-level QD attached to ferromagnetic electrodes and study electrical and heat currents generated due to temperature gradient and applied bias voltage. The non-equilibrium Green function formalism is used to investigate non-linear transport and spin effects are discussed.

\footnotetext{
* corresponding author; e-mail: wierzba@if.pw.edu.pl
}

\section{Model}

Electrical and heat currents flowing through the system in a presence of bias voltage $V_{\mathrm{b}}$ and temperature gradient are calculated according to the following formulae:

$$
\begin{aligned}
& I=\frac{e}{\hbar} \int \frac{\mathrm{d} E}{2 \pi} T(E)\left(f_{\mathrm{L}}-f_{\mathrm{R}}\right), \\
& I_{Q}=\frac{1}{\hbar} \int \frac{\mathrm{d} E}{2 \pi} T(E)(E-\bar{\mu})\left(f_{\mathrm{L}}-f_{\mathrm{R}}\right),
\end{aligned}
$$

where $f_{\beta} \beta=\mathrm{L}, \mathrm{R}$ ) denotes the Fermi-Dirac distribution function $f_{\beta}=\left[1+\exp \left(\left(E-\mu_{\beta}\right) / k T_{\beta}\right)\right]^{-1}$ corresponding to the $\beta$ electrode with electrochemical potential $\mu_{\mathrm{R}, \mathrm{L}}=$ $\mu_{0} \pm \frac{1}{2} V_{\mathrm{b}}$. Here $\mu_{0}$ is the Fermi energy of the system in equilibrium and $T_{\beta}$ denotes temperature of the lead $\beta$. In expression $(2) \bar{\mu}=\frac{1}{2}\left[\mu_{\mathrm{L}}+\mu_{\mathrm{R}}+\operatorname{sgn}\left(f_{\mathrm{L}}-f_{\mathrm{R}}\right)\left(\mu_{\mathrm{L}}-\mu_{\mathrm{R}}\right)\right]$. Such a relation ensures that the heat is carried by electrons whose energies are higher than the chemical potential of the drain electrode [10]. In the above formulae $T(E)$ denotes transmission coefficient.

The transmission is calculated for QD attached to ferromagnetic electrodes. The system is described by the following Hamiltonian: $H=H_{\mathrm{D}}+H_{\mathrm{e}}+H_{\mathrm{T}}$ with

$$
H_{\mathrm{D}}=\sum_{\sigma=\uparrow, \downarrow} \epsilon_{0} d_{\sigma}^{\dagger} d_{\sigma}+U d_{\uparrow}^{\dagger} d_{\uparrow} d_{\downarrow}^{\dagger} d_{\downarrow}
$$

corresponding to the dot with energy level $\epsilon_{0}$ whose position can be shifted by the gate voltage. The electronelectron correlations on the dot are described by the second term in $H_{\mathrm{D}}$ with correlation parameter $U$. The second term in the main Hamiltonian $H_{\mathrm{e}}$ corresponds to free-electron gas in electrodes. In general, magnetic moments in the leads can be non-collinear and form an angle $\theta$. The spin orientation in the dot is determined according to the global reference system, while the local reference frames, determined by the local magnetization orientations, are taken for the leads [8]. $H_{\mathrm{T}}$ describes tunnel effects between the dot and electrodes and is taken 
in the form given in Ref. [8]. Elements of tunneling matrix, which appear in $H_{\mathrm{T}}$ depend on the angle $\theta$.

To determine the transmission the non-equilibrium Green function approach is used within the Hartree-Fock approximation. Then, $T(E)$ can be expressed as follows:

$$
T(E)=\frac{1}{2} \operatorname{Tr}\left(\hat{\Gamma}^{\mathrm{L}} \hat{G}^{\mathrm{r}} \hat{\tilde{\Gamma}}^{\mathrm{R}} \hat{G}^{\mathrm{a}}+\hat{\Gamma}^{\mathrm{R}} \hat{G}^{\mathrm{r}} \hat{\tilde{\Gamma}}^{\mathrm{L}} \hat{G}^{\mathrm{a}}\right),
$$

where $\hat{G}^{\mathrm{r}}, \hat{G}^{\mathrm{a}}$ are the retarded (advanced) Green functions written in a matrix form in the spin space and determined from Dyson's equation. $\hat{\Gamma}^{\beta}$ are matrices whose elements describe spin-dependent couplings to electrodes and $\hat{\tilde{\Gamma}}^{\beta}=\hat{\Gamma}^{\beta}\left(\hat{\Gamma}^{\mathrm{L}}+\hat{\Gamma}^{\mathrm{R}}\right)^{-1} \hat{\Gamma}_{\text {eff }}$.

The matrix $\hat{\Gamma}_{\text {eff }}$ is determined from the self-energy of the interacting system calculated from Dyson's equation [8].

\section{Numerical results and conclusions}

Electrical and heat currents flowing through the dot under the bias voltage and temperature gradient are calculated according to formulae (1) and (2), respectively. Temperature of the right electrode is kept constant and equal to $k T_{\mathrm{R}}=0.1 \mathrm{meV}$, whereas temperature of the left one can be varied. The bias voltage is applied symmetrically to the system and for positive $V_{\mathrm{b}}$ the chemical potential of the right electrode is shifted towards higher energy values, so the bias voltage moves electrons in the opposite direction than the temperature gradient. $\mathrm{Nu}-$ merical calculations are performed for the following parameters: coupling strength to electrodes $\Gamma=0.15 \mathrm{meV}$, $U=2 \mathrm{meV}$, polarization factor in the leads $p=0.8$. Position of the dot energy level can be shifted with the use of gate voltage $V_{\mathrm{g}}$. Heat and charge currents calculated for two situations with magnetic moments parallel in both electrodes ( $\mathrm{P}$ configuration, $\theta=0$ ) and antiparallel (AP configuration, $\theta=\pi$ ) are presented in Fig. 1 . The currents flow under the bias voltage applied to the system, whereas the temperature of the left electrode is constant and equal to $k T_{\mathrm{L}}=0.4 \mathrm{meV}$. Let us note that due to temperature difference both currents are strongly asymmetric with respect to bias polarisation reversal. When $V_{\mathrm{b}}=0$ electrons move from the left to the right under the temperature gradient carrying the heat and the charge. This electron flow is blocked in a presence of the positive bias voltage applied to the system. If $V_{\mathrm{b}}$ is large enough electrons are forced to move in the opposite direction, against the temperature gradient, which corresponds to negative $I_{Q}$. Then, the system works as a cooling device. Both currents strongly depend on the configuration and are much larger when the moments are parallel. Moreover, a slightly higher voltage $V_{\mathrm{b}}$ is required to block the current in AP configuration than in $\mathrm{P}$ one. Thereby, the thermoelectric effect is more pronounced in AP configuration and the generated thermopower is greater than in $\mathrm{P}$ configuration. This conclusion is consistent with results obtained in the linear transport regime [8].

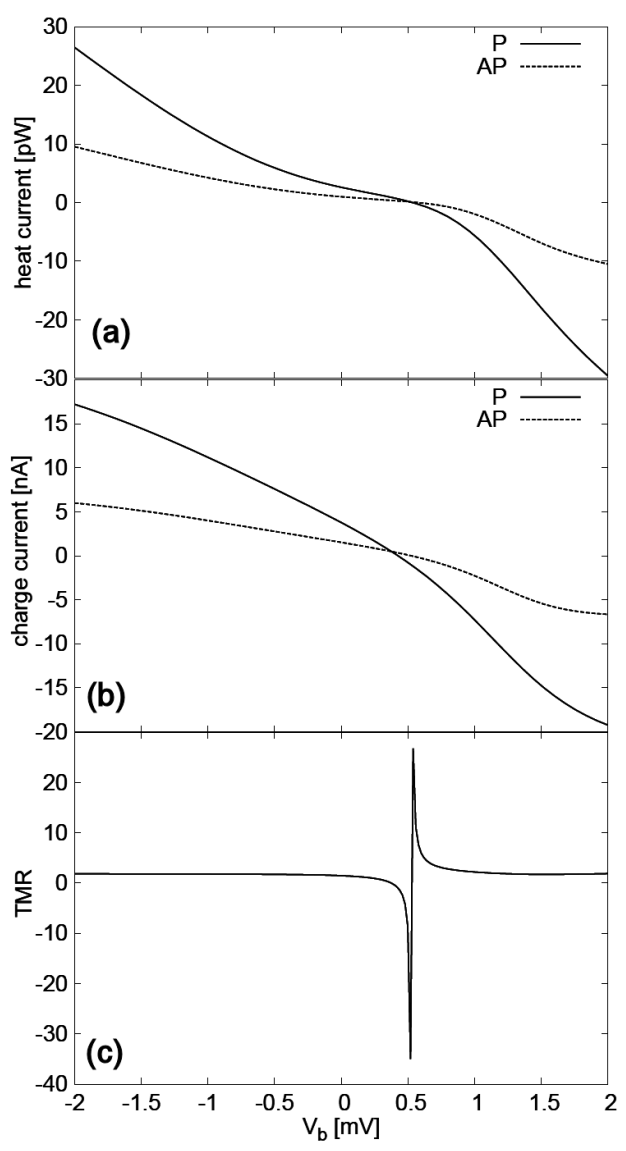

Fig. 1. (a) Heat and (b) charge currents as well as (c) TMR ratio as a function of bias voltage calculated in a presence of temperature difference with $k T_{\mathrm{L}}=0.4 \mathrm{meV}$, $k T_{\mathrm{R}}=0.1 \mathrm{meV}$. Other parameters: $V_{\mathrm{g}}=0.66 \mathrm{meV}$, $\Gamma=0.15 \mathrm{meV}, U=2 \mathrm{meV}, p=0.8$.

Tunnel magnetoresisance (TMR) defined in a way $\mathrm{TMR}=[I(P)-I(A P)] / I(A P)$ is calculated as a function of bias voltage in a presence of temperature difference is also presented in Fig. 1 . The ratio is mostly positive indicating on the normal TMR effect, but it strongly changes in a region of small positive voltage, where flow of electrons under temperature gradient is blocked by the bias voltage applied. In this region TMR can assume large positive and negative values. Negative TMR is a consequence of the fact that in the parallel configuration the blocking voltage is lower than in antiparallel one. Thereby, in a small region of $V_{\mathrm{b}}$ current in both configurations can flow in opposite directions leading to negative TMR.

Electrical and heat currents in a presence of constant bias voltage $V_{\mathrm{b}}=0.2 \mathrm{meV}$ calculated as a function of temperature in the left electrode are presented in Fig. 2 for several configurations of magnetic moments. One can see that the currents almost linearly increase with temperature and strongly depend on the angle. Both currents are the largest in the parallel configuration. Then, TMR ratio is positive and monotonically decreases with $T_{\mathrm{L}}$. 


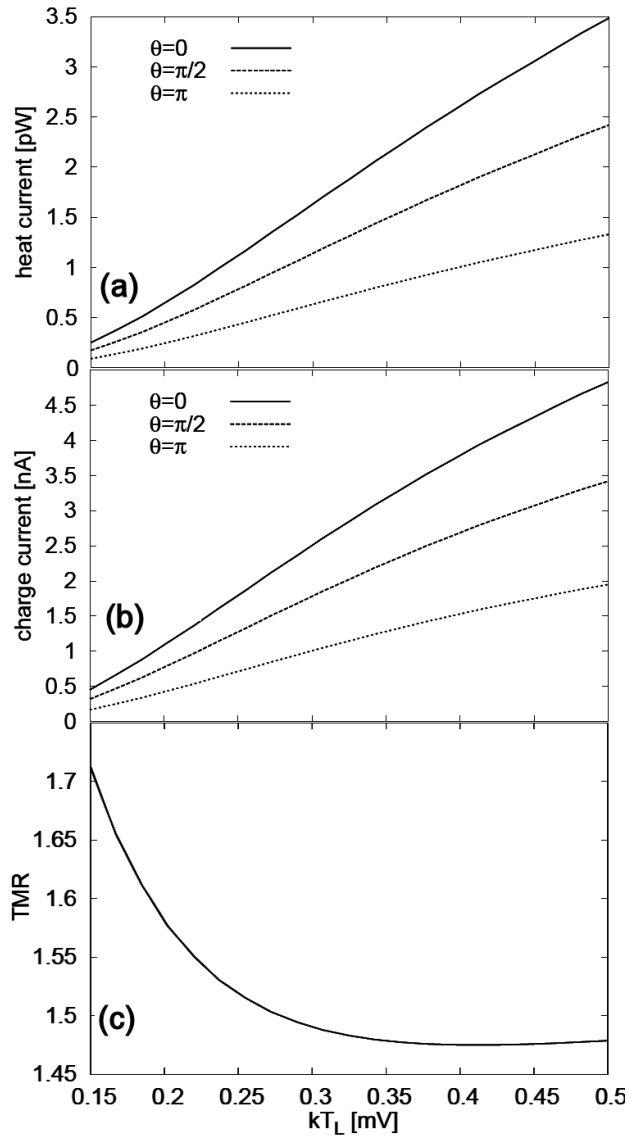

Fig. 2. (a) Heat and (b) charge currents as well as (c) TMR ratio as a function of temperature of the left electrode calculated for indicated values of $\theta$ and $V_{\mathrm{b}}=0.2 \mathrm{meV}, k T_{\mathrm{R}}=0.1 \mathrm{meV}$. Other parameters are the same as in Fig. 1.

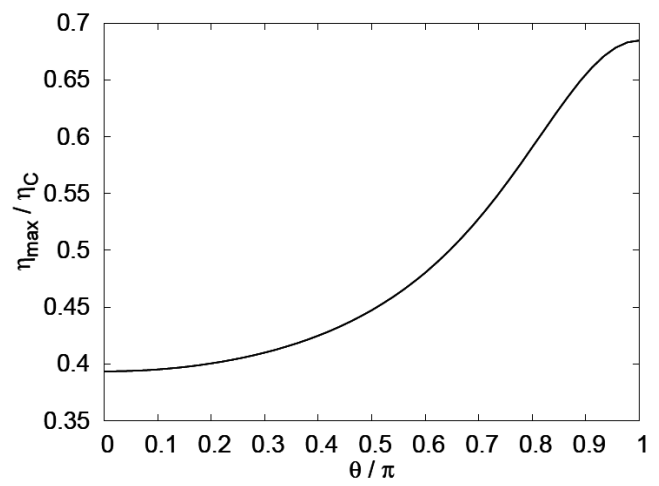

Fig. 3. Maximal efficiency of the system as function of angle $\theta$ for $k T_{\mathrm{L}}=0.4 \mathrm{meV}, k T_{\mathrm{R}}=0.1 \mathrm{meV}$ and positive gate voltage. Other parameters: $\Gamma=0.15 \mathrm{meV}, U=$ $2 \mathrm{meV}, p=0.8$.
Next, the efficiency of the system is investigated. In the non-linear transport regime it can be calculated as: $\eta=P / I_{Q}$ with $P=I V_{\mathrm{b}}$ being the output power $[3,4]$. For a given temperature gradient the output power is different from zero only in the region of small bias voltages, which do not exceed the voltage blocking the charge current. Power $P$ as well as efficiency $\eta$ strongly depend on the gate voltage. The maximal efficiency of the system is calculated in the region of positive gate voltages which corresponds to the situation with dot energy level lying above the Fermi level in equilibrium. As presented in Fig. 3, $\eta_{\max }$ considerably increases when the configuration of magnetic moments in the leads is varied from the parallel to antiparallel one.

At relatively high temperature difference with $k T_{\mathrm{L}}=$ $0.4 \mathrm{meV}$, the high efficiency of the order of 0.7 of efficiency in the Carnot cycle can be achieved in antiparallel configuration (Fig. 3). Thereby, under such conditions the system could work as an efficient energy converter, with a quite considerable output power.

\section{References}

[1] A.A. Staring, L.W. Molenkamp, B.W. Alphenaar, H. van Houten, O.J.A. Buyk, M.A.A. Mabesoone, C.W.J. Beenakker, C.T. Foxon, Europhys. Lett. 22, 57 (1993).

[2] P. Reddy, S.Y. Jang, R.A. Segalman, A. Mujaundar, Science 315, 1568 (2007).

[3] M. Leijnse, M.R. Wegewijs, K. Flensberg, Phys. Rev. B 82, 045412 (2010).

[4] N. Nakpathomkun, H.Q. Xu, H. Linke, Phys. Rev. B 82, 235428 (2010).

[5] K. Uchida, S. Takahashi, K. Harii, J. Ieda, W. Koshibae, K. Ando, S. Maekawa, E. Saitoh, Nature 455, 778 (2008).

[6] M. Hatami, G.E.W. Bauer, Q. Zhang, P.J. Kelly, Phys. Rev. B 79, 174426 (2009).

[7] Y. Dubi, M. Di Ventra, Phys. Rev. B 79, 081302 (2009).

[8] R. Świrkowicz, M. Wierzbicki, J. Barnaś, Phys. Rev. B 80, 195409 (2009).

[9] M. Wierzbicki, R. Świrkowicz, Phys. Rev. B 82, 165334 (2010).

[10] C.D. Mahan, Many-Particle Physics, Plenum, New York 2000. 